\title{
The Gould's Belt Distances Survey (GOBELINS). V. Distances and Kinematics of the Perseus Molecular Cloud
}

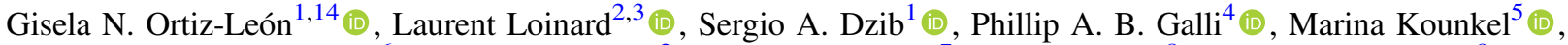 \\ Amy J. Mioduszewski ${ }^{6}$, Luis F. Rodríguez ${ }^{2}$ (i), Rosa M. Torres ${ }^{7}$, Lee Hartmann ${ }^{8}$ (1) , Andrew F. Boden ${ }^{9}$, \\ Neal J. Evans, II $^{10}$, Cesar Briceño ${ }^{11}$ (D), and John J. Tobin ${ }^{12,13}$ (i) \\ ${ }^{1}$ Max Planck Institut für Radioastronomie, Auf dem Hügel 69, D-53121 Bonn, Germany; gortiz@mpifr-bonn.mpg.de \\ ${ }^{2}$ Instituto de Radioastronomía y Astrofísica, Universidad Nacional Autónoma de Mexico, Morelia 58089, México \\ ${ }^{3}$ Instituto de Astronomía, Universidad Nacional Autónoma de México, Apartado Postal 70-264, 04510 Ciudad de México, México \\ ${ }^{4}$ Instituto de Astronomia, Geofísica e Ciências Atmosféricas, Universidade de São Paulo, Rua do Matão 1226, Cidade Universitária, São Paulo, Brazil \\ ${ }^{5}$ Department of Physics and Astronomy, Western Washington University, 516 High Street, Bellingham, WA 98225, USA \\ ${ }^{6}$ National Radio Astronomy Observatory, Domenici Science Operations Center, 1003 Lopezville Road, Socorro, NM 87801, USA \\ ${ }^{7}$ Centro Universitario de Tonalá, Universidad de Guadalajara, Avenida Nuevo Periférico No. 555, Ejido San José Tatepozco, C.P. 48525, Tonalá, Jalisco, México \\ ${ }^{8}$ Department of Astronomy, University of Michigan, 500 Church Street, Ann Arbor, MI 48105, USA \\ ${ }^{9}$ Division of Physics, Math and Astronomy, California Institute of Technology, 1200 East California Boulevard, Pasadena, CA 91125, USA \\ ${ }^{10}$ Department of Astronomy, The University of Texas at Austin, 2515 Speedway, Stop C1400, Austin, TX 78712-1205, USA \\ 11 Cerro Tololo Interamerican Observatory, Casilla 603, La Serena, Chile
${ }^{12}$ Homer L. Dodge Department of Physics and Astronomy, University of Oklahoma, 440 W. Brooks Street, Norman, OK 73019, USA \\ ${ }^{13}$ Leiden Observatory, PO Box 9513, NL-2300 RA Leiden, The Netherlands \\ Received 2018 May 29; revised 2018 August 1; accepted 2018 August 10; published 2018 September 24
}

\begin{abstract}
We derive the distance and structure of the Perseus molecular cloud by combining trigonometric parallaxes from Very Long Baseline Array (VLBA) observations, taken as part of the GOBELINS survey and Gaia Data Release 2. Based on our VLBA astrometry, we obtain a distance of $321 \pm 10 \mathrm{pc}$ for IC 348 . This is fully consistent with the mean distance of $320 \pm 26$ measured by Gaia. The VLBA observations toward NGC 1333 are insufficient to claim a successful distance measurement to this cluster. Gaia parallaxes, on the other hand, yield a mean distance of $293 \pm 22$ pc. Hence, the distance along the line of sight between the eastern and western edges of the cloud is $\sim 30 \mathrm{pc}$, which is significantly smaller than previously inferred. We use Gaia proper motions and published radial velocities to derive the spatial velocities of a selected sample of stars. The average velocity vectors with respect to the LSR are $(\bar{u}, \bar{v}, \bar{w})=(-6.1 \pm 1.6,6.8 \pm 1.1,-0.9 \pm 1.2)$ and $(-6.4 \pm 1.0,2.1 \pm 1.4,-2.4 \pm 1.0) \mathrm{km} \mathrm{s}^{-1}$ for IC 348 and NGC 1333, respectively. Finally, our analysis of the kinematics of the stars has shown that there is no clear evidence of expansion, contraction, or rotational motions within the clusters.
\end{abstract}

Key words: astrometry - radiation mechanisms: non-thermal - radio continuum: stars - stars: individual (IC 348 , NGC 1333) - techniques: interferometric

Supporting material: machine-readable tables

\section{Introduction}

The Perseus molecular cloud represents an ideal target for studying the fundamental properties of young stars and their environment since the complex is sufficiently nearby that spatial scales down to $\sim 50$ au are possible to reach with major observing facilities like the Atacama Large Millimeter/ submillimeter Array (ALMA) and the Very Large Array (VLA). Consisting of an elongated chain of dark clouds, Perseus spans over an area of $7^{\circ} \times 3^{\circ}$ in the plane of the sky. The most prominent substructures are Barnard 5 (B5) and IC 348, at the eastern edge and Barnard 1 (B1), NGC 1333, L1448, L1451, and L1455 at the western edge of the complex (see, e.g., Bally et al. 2008 for a comprehensive review). Most of the young stars reside in IC 348 and NGC 1333, which contain about 480 and 200 objects, respectively, with ages of 1-3 Myr (Luhman et al. 2016), mainly identified from optical and near-IR surveys. The protostellar content within Perseus, on the other hand, has been probed with observations at mid-IR (Spitzer; Enoch et al. 2009), far-IR (Herschel; Sadavoy et al. 2014), submillimeter (JCMT; Sadavoy et al. 2010), and radio

\footnotetext{
${ }^{14}$ Humboldt Fellow.
}

(VLA; Tobin et al. 2016; Tychoniec et al. 2018) wavelengths. A total of 94 Class 0/I protostars and flat-spectrum/Class II objects are known to populate the entire cloud (Tobin et al. 2016).

Multiple measurements of the distance to the individual clouds in Perseus have been performed in the past. These measurements suggest that there is a distance gradient across the cloud, with values in the range from 212 to $260 \mathrm{pc}$ for the western component of the cloud (Cernis 1990; Hirota et al. 2008, 2011; Lombardi et al. 2010; Schlafly et al. 2014) and 260-315 pc for the eastern component (Cernis 1993; Lombardi et al. 2010; Schlafly et al. 2014). Direct measurement of distances via the trigonometric parallax have been obtained for only a few sources in these regions. Based on Very Long Baseline Interferometry (VLBI) observations of $\mathrm{H}_{2} \mathrm{O}$ masers associated with two young stellar objects (YSOs) in NGC 1333 and L1448, Hirota et al. $(2008,2011)$ found a distance consistent with $235 \mathrm{pc}$ for both clouds. However, whether or not the gradient in the distance across the whole complex is significant remains inconclusive since the distance uncertainties on individual lines of sight are large (typically $\sim 10 \%-20 \%$ for photometric distances), and the number of sources with available direct distance measurements is small. 
In the past few years, we have used the Very Long Baseline Array (VLBA) to measure the trigonometric parallax of several tens of young stars in nearby star-forming regions (Kounkel et al. 2017; Ortiz-León et al. 2017a, 2017b; Galli et al. 2018) as part of the Gould's Belt Distances Survey (GOBELINS) project. VLBI has the advantage of being able to detect highly embedded sources, where the extinction by dust obscures the optical light from the stellar objects. Given the high angular resolution provided by the VLBA and the fact that the interstellar material in these regions is transparent to radio waves, parallaxes with an accuracy of $1 \%$ or better are possible for these kind of sources. In addition, parallaxes toward more than 400 stars in Perseus with a limiting magnitude $G=21$ mag and parallax uncertainties $<0.7$ mas have become available during the second Gaia data release (DR2). With this highly accurate astrometric data, we can now investigate the depth of the molecular cloud and the three-dimensional motions of the young stars as well as the global properties of the kinematics of IC 348 and NGC 1333.

We first describe the VLBA observations in Section 2 and the fits to our data in Section 3. Section 4 presents the extraction of the astrometric solutions from the Gaia DR2 catalog. We then use both VLBA and Gaia data to investigate the structure of the Perseus cloud, which is discussed in Section 5. Sections 6.1 and 6.2 present the kinematics of a selected sample of cluster members in IC 348 and NGC 1333. Finally, our conclusions are given in Section 7.

\section{VLBA Observations and Data Reduction}

The target selection for the VLBA survey and observing strategy follows the same procedure described in detail in Ortiz-León et al. (2017b). In summary, we constructed our target sample based on the properties of the radio emission detected with the Very Large Array toward YSOs and YSO candidates in NGC 1333 and IC 348 (Pech et al. 2016). We observed all radio sources associated with YSOs whose radio emission could be detected with the VLBA, i.e., nonthermal sources. In addition, we observed all unidentified sources in the region whose radio properties are consistent with YSOs and have fluxes above the threshold of the GOBELINS observations. In total, 59 sources were observed between 2011 April and 2018 March at $\nu=5.0$ or $8.4 \mathrm{GHz}$ (C and $\mathrm{X}$ band, respectively). The data were recorded in dual-polarization mode with $256 \mathrm{MHz}$ of bandwidth in each polarization, covered by eight separate $32 \mathrm{MHz}$ intermediate-frequency (IF) channels. Each observing session consisted of cycles alternating between the target and J0336+3218. Three additional calibrators were observed every $\sim 50$ minutes to improve the phase calibration. In addition, geodetic-like blocks, consisting of observations of many calibrators over a wide range of elevations, were taken before and after each session. We use AIPS (Greisen 2003) for data inspection, calibration, and imaging, following standard procedures for phase-referencing observations as described in Ortiz-León et al. (2017b).

Out of the 25 sources detected, only 7 are related to YSOs, while the rest turned out to be background objects with negligible motion on the plane of the sky. In this paper, we present a subset of detected YSOs for which we can measure both parallax and proper motions (four sources in total). The other three sources have detections in only 1-2 epochs, which is insufficient to perform the astrometric fits. The dates of these
Table 1

VLBA Observed Epochs

\begin{tabular}{|c|c|c|c|c|}
\hline \multirow{2}{*}{$\begin{array}{l}\text { Project } \\
\text { Code }\end{array}$} & \multirow{2}{*}{$\begin{array}{l}\text { Observation } \\
\text { Date }\end{array}$} & \multicolumn{2}{|c|}{ VLBA Pointing Positions } & \multirow{2}{*}{$\begin{array}{c}\text { Observed } \\
\text { Band }\end{array}$} \\
\hline & & R.A. $\left(\alpha_{2000}\right)$ & Decl. $\left(\delta_{2000}\right)$ & \\
\hline BL175CD & 2012 Sep 04 & 03:44:34.77 & 32:07:43.99 & $\mathrm{X}$ \\
\hline BL175CF & 2012 Sep 07 & 03:45:00.92 & $32: 04: 19.03$ & $\mathrm{X}$ \\
\hline BL175AS & 2013 Mar 22 & 03:44:34.77 & $32: 07: 43.99$ & $\mathrm{X}$ \\
\hline BL175AU & 2013 Apr 18 & 03:45:00.92 & $32: 04: 19.03$ & $\mathrm{X}$ \\
\hline BL175H8 & 2014 Apr 13 & 03:45:00.92 & $32: 04: 19.03$ & $\mathrm{X}$ \\
\hline BL175EF & 2014 Sep 06 & $\begin{array}{l}03: 43: 58.63 \\
03: 44: 21.89 \\
03: 44: 34.77\end{array}$ & $\begin{array}{l}32: 01: 45.64 \\
32: 09: 48.04 \\
32: 07: 43.99\end{array}$ & $X$ \\
\hline BL175CQ & 2014 Sep 13 & 03:45:00.92 & $32: 04: 19.03$ & $\mathrm{X}$ \\
\hline BL175EW & 2015 Apr 26 & 03:45:00.92 & $32: 04: 19.03$ & $\mathrm{X}$ \\
\hline BL175HS & 2015 Oct 20 & 03:45:00.92 & $32: 04: 19.03$ & $\mathrm{X}$ \\
\hline BL175HU & 2015 Oct 24 & $\begin{array}{l}03: 43: 58.63 \\
03: 44: 21.89 \\
03: 44: 34.77\end{array}$ & $\begin{array}{l}32: 01: 45.64 \\
32: 09: 48.04 \\
32: 07: 43.99\end{array}$ & $\mathrm{X}$ \\
\hline BL175I9 & 2016 Apr 07 & 03:45:07.97 & 32:04:01.81 & $\mathrm{C}$ \\
\hline BL175IB & 2016 Apr 11 & $\begin{array}{l}03: 28: 50.00 \\
03: 29: 03.00 \\
03: 29: 20.00\end{array}$ & $\begin{array}{l}31: 30: 00.00 \\
31: 22: 00.00 \\
31: 14: 00.00\end{array}$ & $\mathrm{C}$ \\
\hline BL175ID & 2016 Apr 30 & $\begin{array}{l}03: 44: 25.00 \\
03: 44: 45.00\end{array}$ & $\begin{array}{l}32: 08: 30.00 \\
32: 17: 00.00\end{array}$ & $\mathrm{C}$ \\
\hline BL175IP & 2016 Aug 26 & $\begin{array}{l}03: 28: 50.00 \\
03: 29: 03.00 \\
03: 29: 20.00\end{array}$ & $\begin{array}{l}31: 30: 00.00 \\
31: 22: 00.00 \\
31: 14: 00.00\end{array}$ & $\mathrm{C}$ \\
\hline BL175J6 & 2016 Aug 27 & $\begin{array}{l}03: 44: 25.00 \\
03: 44: 45.00\end{array}$ & $\begin{array}{l}32: 08: 30.00 \\
32: 17: 00.00\end{array}$ & $\mathrm{C}$ \\
\hline BL175J1 & 2016 Oct 11 & 03:45:07.97 & $32: 04: 01.81$ & $\mathrm{C}$ \\
\hline BL175JX & 2017 Apr 24 & 03:45:07.97 & $32: 04: 01.81$ & $\mathrm{C}$ \\
\hline BL175KN & 2017 Oct 06 & 03:45:07.97 & 32:04:01.81 & $\mathrm{C}$ \\
\hline BL175KH & 2017 Oct 07 & $\begin{array}{l}03: 28: 46.49 \\
03: 29: 03.00 \\
03: 29: 20.00\end{array}$ & $\begin{array}{l}31: 29: 43.50 \\
31: 22: 00.00 \\
31: 14: 00.00\end{array}$ & $\mathrm{C}$ \\
\hline BL175KI & 2017 Oct 13 & $\begin{array}{l}03: 44: 25.00 \\
03: 44: 45.00\end{array}$ & $\begin{array}{l}32: 08: 30.00 \\
32: 17: 00.00\end{array}$ & $\mathrm{C}$ \\
\hline BL175KY & 2018 Mar 15 & $\begin{array}{l}03: 29: 10.39 \\
03: 44: 32.59 \\
03: 45: 07.96\end{array}$ & $\begin{array}{l}31: 21: 59.00 \\
32: 08: 42.35 \\
32: 04: 01.75\end{array}$ & $\mathrm{C}$ \\
\hline
\end{tabular}

observations and VLBA pointing positions are given in Table 1.

\section{VLBA Astrometry}

Source positions at individual epochs were extracted by performing two-dimensional Gaussian fits with the AIPS task JMFIT (Table 2). Parallax, $\varpi$, position at median epoch, $\left(\alpha_{0}, \delta_{0}\right)$, and proper motions, $\mu_{\alpha}$ and $\mu_{\delta}$, were fitted to the measured positions by minimizing $\chi^{2}$ in each direction. Systematic errors were added to the statistical errors provided by JMFIT. These errors were obtained by scaling positional uncertainties until the reduced $\chi^{2}$ of the fit becomes equal to 1 . The resulting best-fit parameters are shown in columns (2)-(4) of Table 3 and illustrated in Figure 1. We briefly discuss each source in the following paragraphs.

\subsection{IRAS $03260+3111=2$ MASS $J 03291037+3121591$}

This is a known wide binary system with a separation of 3."62 (Haisch et al. 2004) located in NGC 1333. It has been classified as a Class II object (Gutermuth et al. 2008) of spectral type F5 (Luhman et al. 2016). We simultaneously detected two sources with the VLBA in two epochs, with an angular separation of $\sim 0$ !' 7 . The closer companion was already 
Table 2

VLBA Measured Source Positions

\begin{tabular}{|c|c|c|c|c|}
\hline Julian Day & $\alpha(\mathrm{J} 2000.0)$ & $\sigma_{\alpha}$ & $\delta(\mathrm{J} 2000.0)$ & $\sigma_{\delta}$ \\
\hline \multicolumn{5}{|c|}{ V913PER } \\
\hline 2456374.43416 & 34432.58795488 & 0.00001138 & 32842.372174 & 0.000296 \\
\hline 2457319.91111 & 34432.58879683 & 0.00000899 & 32842.354985 & 0.000213 \\
\hline 2457509.32628 & 34432.58867044 & 0.00001174 & 32842.348552 & 0.000277 \\
\hline 2457628.01584 & 34432.58905351 & 0.00000866 & 32842.348630 & 0.000213 \\
\hline 2458039.88818 & 34432.58918298 & 0.00000718 & 32842.340549 & 0.000184 \\
\hline 2458193.47921 & 34432.58891352 & 0.00000484 & 32842.335871 & 0.000118 \\
\hline 2458209.43523 & 34432.58895319 & 0.00000232 & 32842.335604 & 0.000064 \\
\hline \multicolumn{5}{|c|}{ V918PER } \\
\hline \multicolumn{5}{|l|}{ first source: } \\
\hline 2457319.91152 & 34436.94228029 & 0.00000324 & 32645.414751 & 0.000058 \\
\hline 2457509.32628 & 34436.94223035 & 0.00001212 & 32645.410445 & 0.000356 \\
\hline 2457628.01584 & 34436.94268097 & 0.00001542 & 32645.409728 & 0.000315 \\
\hline 2458039.88818 & 34436.94304360 & 0.00000313 & 32645.401953 & 0.000083 \\
\hline 2458209.43523 & 34436.94289598 & 0.00000619 & 32645.396791 & 0.000161 \\
\hline second source: & & & & \\
\hline 2456174.97590 & 34436.96218751 & 0.00000269 & 32644.952195 & 0.000068 \\
\hline \multicolumn{5}{|c|}{ LRL 11} \\
\hline 2456177.96799 & 34507.96419667 & 0.00000301 & 32401.790487 & 0.000101 \\
\hline 2456761.37584 & 34507.96445493 & 0.00000820 & 32401.776312 & 0.000194 \\
\hline 2456913.95729 & 34507.96488174 & 0.00000705 & 32401.774299 & 0.000246 \\
\hline 2457139.34132 & 34507.96475367 & 0.00000248 & 32401.766255 & 0.000075 \\
\hline 2457315.85692 & 34507.96497603 & 0.00000207 & 32401.763100 & 0.000052 \\
\hline 2457486.39137 & 34507.96476855 & 0.00000214 & 32401.756829 & 0.000062 \\
\hline 2457672.88009 & 34507.96503320 & 0.00000930 & 32401.754586 & 0.000217 \\
\hline 2457868.34505 & 34507.96484623 & 0.00000337 & 32401.748432 & 0.000126 \\
\hline 2458032.89439 & 34507.96512694 & 0.00000938 & 32401.746482 & 0.000227 \\
\hline 2458193.47921 & 34507.96483889 & 0.00000819 & 32401.742014 & 0.000237 \\
\hline 2458209.43523 & 34507.96484894 & 0.00001005 & 32401.741787 & 0.000255 \\
\hline \multicolumn{5}{|c|}{ 2MASS J03291037+3121591 } \\
\hline \multicolumn{5}{|l|}{ first source: } \\
\hline 2457490.44419 & 32910.36879126 & 0.00001335 & 312158.937104 & 0.000281 \\
\hline 2457627.07078 & 32910.36934100 & 0.00001221 & 312158.932573 & 0.000302 \\
\hline 2458033.95677 & 32910.36911952 & 0.00000841 & 312158.925023 & 0.000144 \\
\hline \multicolumn{5}{|l|}{ second source: } \\
\hline 2457490.44419 & 32910.42062181 & 0.00000777 & 312159.032952 & 0.000176 \\
\hline 2458033.95677 & 32910.42184192 & 0.00001568 & 312159.017736 & 0.000187 \\
\hline 2458193.47921 & 32910.42173882 & 0.00000477 & 312159.011139 & 0.000096 \\
\hline 2458209.43523 & 32910.42183461 & 0.00002250 & 312159.011471 & 0.000280 \\
\hline
\end{tabular}

Table 3

Astrometric Solutions of the VLBA-detected Sources and Their Counterparts in the Gaia DR2 Catalog

\begin{tabular}{|c|c|c|c|c|c|c|c|c|}
\hline \multirow{2}{*}{$\begin{array}{l}\text { Name } \\
\text { (1) }\end{array}$} & \multicolumn{4}{|c|}{ VLBA } & \multicolumn{4}{|c|}{ Gaia } \\
\hline & $\begin{array}{c}\text { Parallax } \\
\text { (mas) } \\
\text { (2) }\end{array}$ & $\begin{array}{c}\text { Distance } \\
\text { (pc) } \\
\text { (3) }\end{array}$ & $\begin{array}{c}\mu_{\alpha} \cos \delta \\
\left(\operatorname{mas~yr}^{-1}\right) \\
(4)\end{array}$ & $\begin{array}{c}\mu_{\delta} \\
\left(\operatorname{mas~yr}^{-1}\right) \\
(5)\end{array}$ & $\begin{array}{c}\text { Parallax } \\
\text { (mas) } \\
\text { (6) }\end{array}$ & $\begin{array}{c}\text { Distance }^{a} \\
\text { (pc) } \\
\text { (7) }\end{array}$ & $\begin{array}{c}\mu_{\alpha} \cos \delta \\
\left(\operatorname{mas~yr}^{-1}\right) \\
(8)\end{array}$ & $\begin{array}{c}\mu_{\delta} \\
\left(\operatorname{mas~yr}^{-1}\right) \\
(9)\end{array}$ \\
\hline IRAS $03260+3111$ & $3.136 \pm 0.152$ & $319_{-15}^{+16}$ & $7.973 \pm 0.083$ & $-11.257 \pm 0.121$ & $\cdots$ & $\cdots$ & $\cdots$ & $\cdots$ \\
\hline V913 Per & $3.119 \pm 0.104$ & $321_{-10}^{+11}$ & $2.458 \pm 0.047$ & $-7.272 \pm 0.133$ & $3.708 \pm 0.262$ & $270_{-18}^{+21}$ & $5.039 \pm 0.482$ & $-7.111 \pm 0.281$ \\
\hline V918 Per & $3.129 \pm 0.512$ & $320_{-45}^{+63}$ & $4.857 \pm 0.335$ & $-6.750 \pm 0.488$ & $1.852 \pm 0.333$ & $549_{-94}^{+140}$ & $-3.321 \pm 0.602$ & $-9.831 \pm 0.439$ \\
\hline LRL 11 & $2.680 \pm 0.076$ & $373_{-10}^{+11}$ & $2.37 \pm 0.08$ & $-8.271 \pm 0.160$ & $2.665 \pm 0.117$ & $372_{-16}^{+17}$ & $1.814 \pm 0.214$ & $-9.807 \pm 0.123$ \\
\hline
\end{tabular}

Note.

a These values were taken from the distance catalog available from the Gaia TAP service of the Astronomisches Rechen Institut (ARI; Bailer-Jones et al. 2018). 

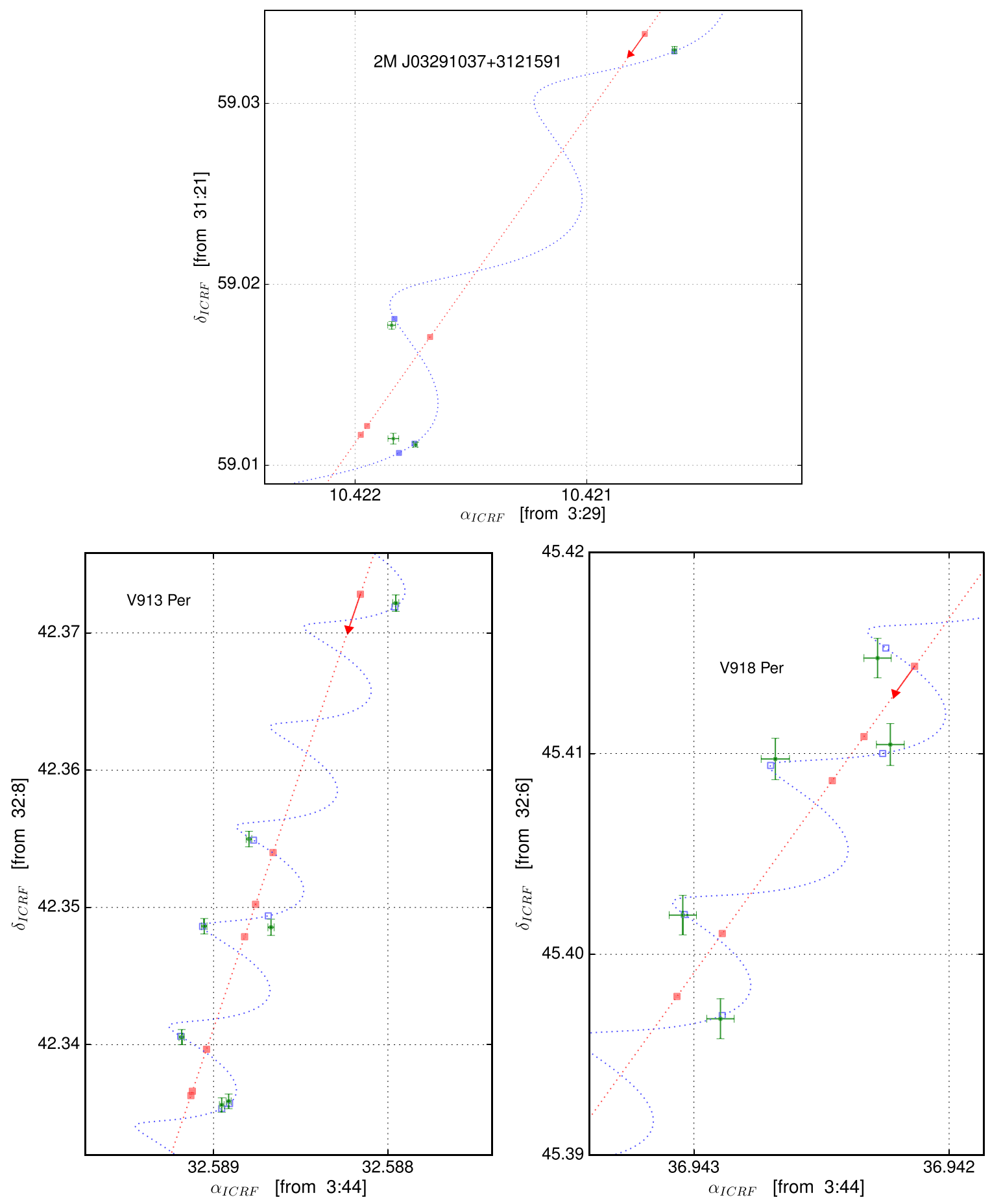

Figure 1. Astrometric fits to VLBA data. Observed positions and expected positions from the fits are shown as green dots and blue open squares, respectively. The blue dotted line is the fitted model, and the red line is the model with the parallax signature removed. The red squares mark the position of the sources from the model without parallax. The arrows show the the direction of position change over time.

seen by Connelley et al. (2008), who found a binary separation of 0 ". 55 in the near-IR. The source is thus a hierarchical triple system. The brightest IR component corresponds to the western radio source seen in our maps.

Given the scarcity of the data from the only five epochs available, the fit including orbital motions does not converge to reliable parameters, and the corresponding uncertainties are large. We thus fit only parallax and proper motions separately to each source and adopt the resulting parameters from the fit to the eastern component, which has four detected epochs.

\section{2. $\mathrm{V} 913 \mathrm{Per}$}

This source is located in IC 348. It is a Class III star of spectral type M2.5 (Luhman et al. 2016). It has been detected in seven epochs, which we used for the derivation of the astrometric parameters. The derived parallax has an uncertainty of $3.3 \%$.

\section{3. $\mathrm{V} 918 \mathrm{Per}$}

Also located in IC 348, this source is a Class II/III object (Alexander \& Preibisch 2012; Young et al. 2015) with a 
Table 4

Orbital Solutions for LRL 11

\begin{tabular}{lc}
\hline \hline Parameter & Best-fit Solution \\
\hline$P$ (year) & $6.3 \pm 0.4$ \\
$a$ (mas) & $2.73 \pm 0.16$ \\
$T_{P}(\mathrm{JD})$ & $2458942 \pm 208$ \\
$e$ & $0.147 \pm 0.078$ \\
$\omega(\mathrm{deg})$ & $291.1 \pm 19.8$ \\
$i(\mathrm{deg})$ & $49.1 \pm 6.8$ \\
$\Omega(\mathrm{deg})$ & $84.4 \pm 8.5$ \\
\hline
\end{tabular}

spectral type of G3 (Luhman et al. 2016). Two sources have been detected in our maps in alternative epochs. One source was only seen in the first epoch, while the other source has been detected in five epochs. We fit only the astrometric parameters to these five epochs.

\subsection{LRL 11}

This source is a Class III star with a spectral type of G4 (Luhman et al. 2016) located in IC 348 as well. The model including only parallax and proper motions produces a poor fit to the data. We investigate if the source motion can be reproduced by adding an orbital component due to the possibility that the source is a binary system. The fit that includes orbital motions does indeed reproduce the measured source positions. We found that the two methods we have used in the past to fit binaries (cf. Kounkel et al. 2017; Galli et al. 2018) yield different solutions for the orbital elements. This means that our data are not good enough to constrain the orbit, so it should be taken somewhat cautiously. On the other hand, the parallax and proper motions from the two methods agree within $2 \sigma$. We give in Table 4 the best-fit solution obtained from the Markov Chain Monte Carlo method (Galli et al. 2018), which is illustrated in Figure 2.

\section{Gaia Data}

With the recent release of Gaia DR2 (Gaia Collaboration et al. 2016, 2018; Lindegren et al. 2018), astrometric data for objects with $G<21 \mathrm{mag}$ have become available. We will use this wealth of new data to assess further the distance to Perseus.

As discussed in Section 1, the most complete catalog to date of young members in IC 348 and NGC 1333 has been compiled by Luhman et al. (2016). This catalog contains 478 and 203 stars in IC 348 and NGC 1333, respectively, for which memberships were confirmed from optical and near-IR spectroscopy. We performed a cross match of the young stars' positions against the Gaia DR2 catalog using a search radius of $1^{\prime \prime}$. The source coordinates in the catalog of Luhman et al. (2016) were either taken from the 2MASS Point Source Catalog (which have a positional accuracy of $0.1-0.13$ ) or measured from previous photometric infrared surveys, where allowed positional shifts are up to $\sim 1^{\prime \prime}$ (Alves de Oliveira et al. 2013). The radial velocity catalogs we will use in Section 6.2 have been constructed from a variety of published $\mathrm{X}$-ray, optical, and mid-infrared surveys, where positional errors range from 0 " 3 to $1^{\prime \prime}$. Thus, the choice of a match radius of $1^{\prime \prime}$ allows us to take into account the different uncertainties from the various surveys. In total, 351 and 90 stars, in IC 348

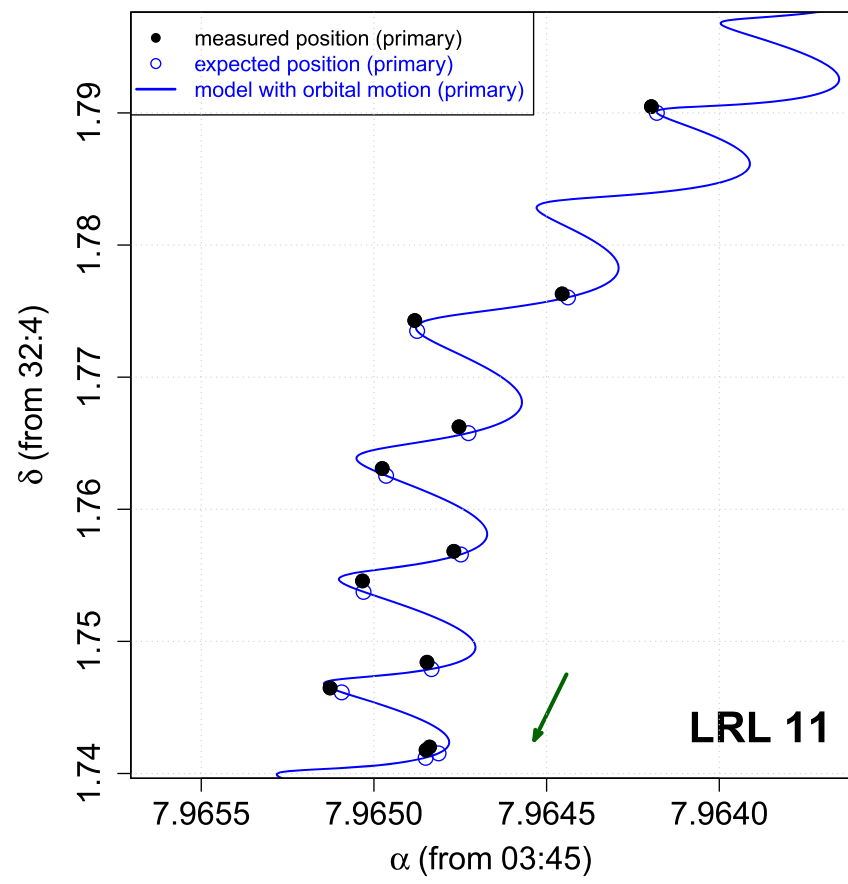

Figure 2. Astrometric fit to VLBA data taken toward LRL 11 including orbital motion. Observed positions and expected positions from the fits are shown as black filled and blue open dots, respectively. The blue line is the fitted model.

and NGC 1333, respectively, have five astrometric parameter solutions.

Three of our VLBA-detected sources appear in the Gaia DR2 catalog. Their astrometric solutions are given in columns (5)-(7) of Table 3. The difference between VLBA and Gaia parallaxes is 0.589 , 1.277, and 0.015 mas for V913 Per, V918 Per, and LRL 11. The proper motions are remarkably different mainly in the R.A. direction. This discrepancy is expected for the binary systems, V918 Per and LRL 11, since all Gaia sources have been treated as single stars in DR2. We argue that the discrepancy in the astrometric solutions for V913 Per can be attributed to systematic errors present in Gaia DR2. The magnitude of these systematic

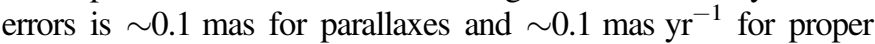
motions (Luri et al. 2018). In addition, a parallax zero-point offset of -0.03 mas, corresponding to the mean parallax of sources identified as quasars, should be also taken into account (Lindegren et al. 2018). If the systematic errors are added quadratically to the quoted uncertainties in Gaia DR2 catalog, then the parallax of V913 Per agrees within $2 \sigma$ and the proper motion in declination within $1 \sigma$. However, the proper motion in right ascension still disagrees by $\sim 5 \sigma$. For this particular source, the quantities astrometric_excess_noise and astrometric_excess_noise_sig, given in the Gaia archive, have values of 1.1 mas and 187.6, respectively. These parameters represent the excess noise of the source and its significance, which measure the difference between the observations and the best-fitting astrometric model. Values of astrometric_excess_noise $>0$ mas (with astrometric_excess_ noise_sig $>2$ ) indicate that the residuals of the fit to the Gaia data are larger than expected due to modeling and calibration errors. Other VLBA sources we have monitored in Orion and Taurus (Kounkel et al. 2017; Galli et al. 2018) show an agreement in proper motion better than $2 \sigma$. It is doubtful that our VLBA data are affected by systematic effects. 

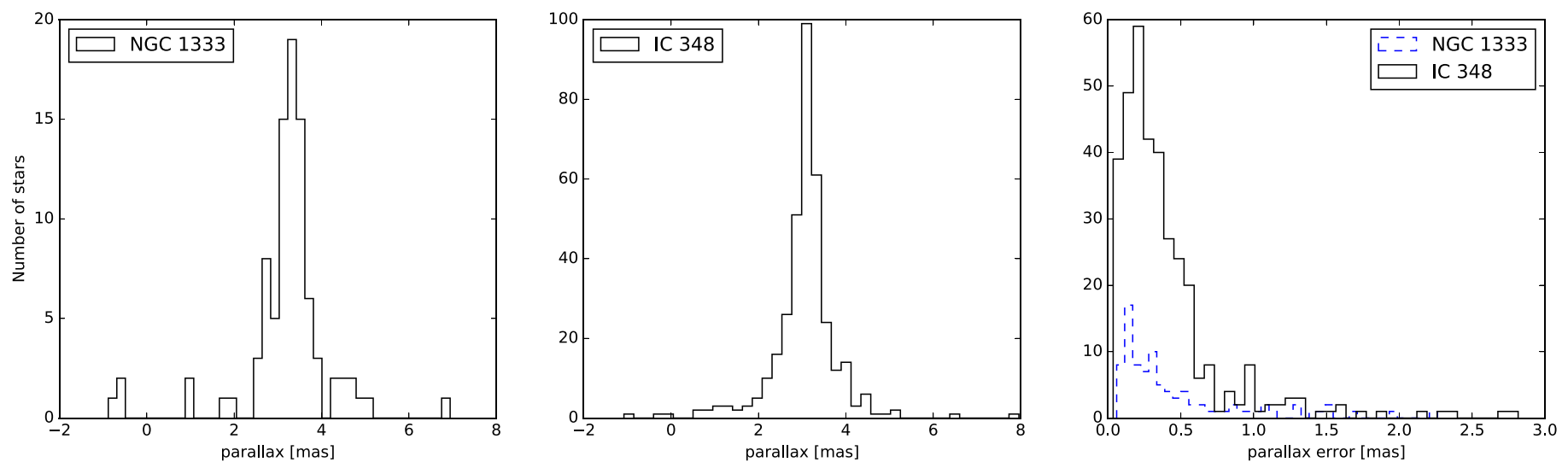

Figure 3. Distributions of parallaxes and their uncertainties measured by Gaia toward Perseus.

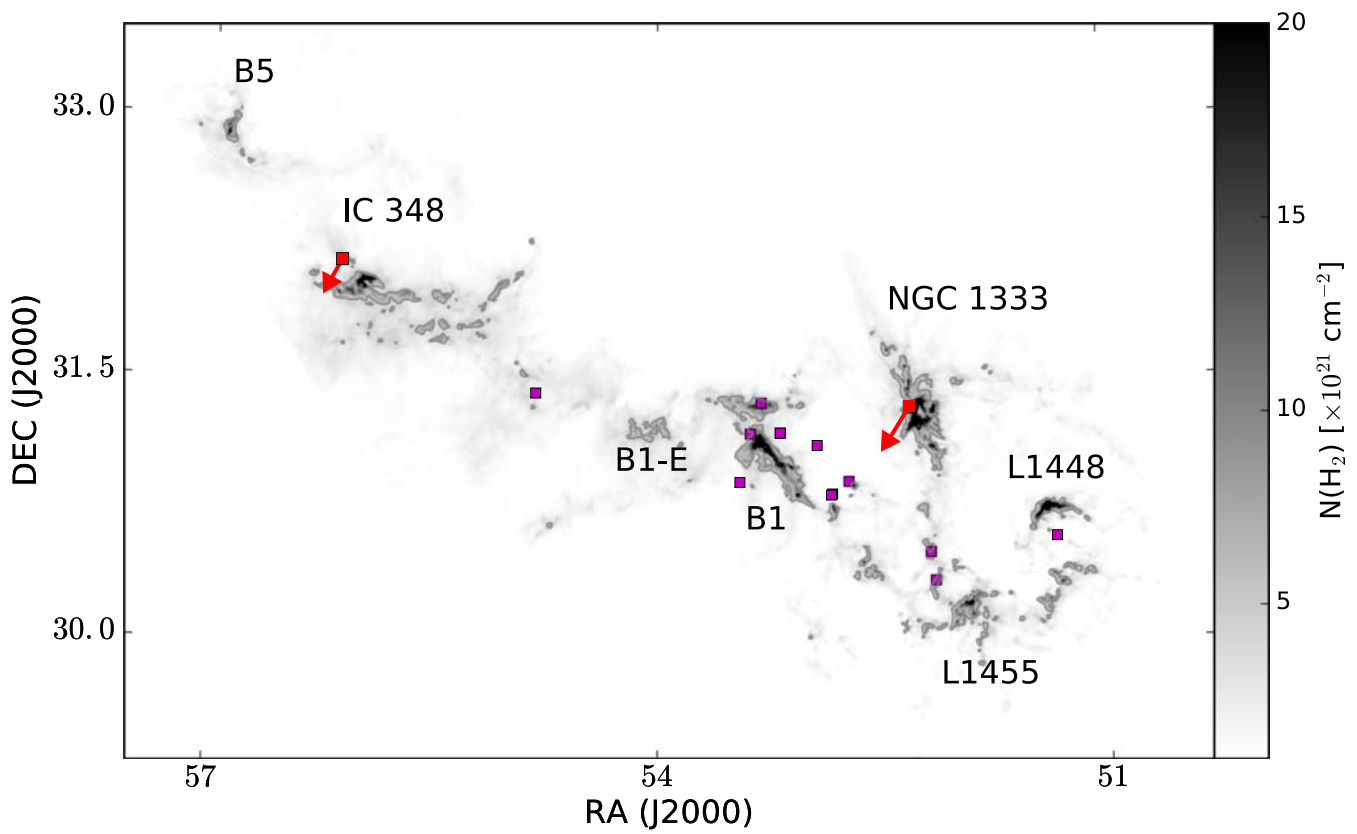

Figure 4. Large-scale column density map of the Perseus molecular cloud from the Herschel Gould Belt survey (André et al. 2010; Sadavoy et al. 2014). The red arrows show the mean of the measured proper motions in IC 348 and NGC 1333 (see Section 6.1). The origin of the arrows is at the mean position of the selected sample of stars described in Section 6.1. The magenta squares are other YSO candidates across Perseus with five astrometric solutions in the Gaia DR2 catalog.

\section{Structure of Perseus}

We show in Figure 3 the distributions of Gaia parallaxes and parallax uncertainties for NGC 1333 and IC 348. The parallax uncertainties have median values of 0.30 mas in both cases. The weighted mean of these parallaxes is $\varpi=3.38 \pm 0.02$ mas with a weighted standard deviation of $\sigma_{\varpi}=0.32$ mas for NGC 1333 and $\varpi=3.09 \pm 0.01$ mas with $\sigma_{\varpi}=0.26$ mas for IC 348. Inverting the weighted mean parallaxes (after correcting for the parallax zero-point shift of $-30 \mu$ as) yields a distance of $d=294 \mathrm{pc}$ with a standard deviation of $\sigma_{d}=28 \mathrm{pc}$ for NGC 1333 and $d=321 \mathrm{pc}$ with $\sigma_{d}=27 \mathrm{pc}$ for IC 348 . If we remove the stars with parallaxes outside the core of the parallax distribution (i.e., with $\varpi<1.5$ and $>6$ mas in NGC 1333 and $\varpi<0.4$ and $>6$ mas in IC 348 ), the mean parallaxes give $d=293 \pm 22 \mathrm{pc}$ for NGC 1333 and $d=320 \pm 26 \mathrm{pc}$ for IC 348 , where the quoted errors correspond to the standard deviation. In each cluster, the data have median of parallax uncertainties larger than the standard deviation of the whole distribution. This means that the parallax dispersion is not
Table 5

Gaia Parallaxes and Proper Motions of YSO Candidates outside NGC 1333 and IC 348

\begin{tabular}{|c|c|c|c|}
\hline $\begin{array}{l}\text { Spitzer Source } \\
\text { Name } \\
\text { (1) }\end{array}$ & $\begin{array}{l}\text { Parallax } \\
\text { (mas) } \\
(2)\end{array}$ & $\begin{array}{c}\mu_{\alpha} \cos \delta \\
\left(\operatorname{mas~yr}^{-1}\right) \\
(3)\end{array}$ & $\begin{array}{c}\mu_{\delta} \\
\left(\operatorname{mas~yr}^{-1}\right) \\
(4)\end{array}$ \\
\hline J032519.5+303424 & $0.68 \pm 0.43$ & $1.21 \pm 0.72$ & $0.08 \pm 0.47$ \\
\hline J032835.0+302009 & $1.9 \pm 1.98$ & $5.7 \pm 2.83$ & $-9.82 \pm 1.94$ \\
\hline J032842.4+302953 & $3.62 \pm 0.14$ & $6.36 \pm 0.26$ & $-9.75 \pm 0.15$ \\
\hline $\mathrm{J} 033052.5+305417$ & $3.17 \pm 0.3$ & $7.09 \pm 0.37$ & $-7.79 \pm 0.31$ \\
\hline J033118.3+304939 & $3.32 \pm 0.08$ & $7.27 \pm 0.12$ & $-7.8 \pm 0.09$ \\
\hline J033120.1+304917 & $3.9 \pm 0.24$ & $7.86 \pm 0.35$ & $-8.25 \pm 0.24$ \\
\hline J033142.4+310624 & $3.67 \pm 0.17$ & $7.91 \pm 0.25$ & $-6.56 \pm 0.17$ \\
\hline J033241.6+311044 & $4.05 \pm 0.84$ & $7.58 \pm 1.59$ & $-8.63 \pm 0.84$ \\
\hline J033241.7+311046 & $2.78 \pm 0.24$ & $7.65 \pm 0.49$ & $-7.65 \pm 0.26$ \\
\hline J033312.8+312124 & $2.2 \pm 0.57$ & $6.12 \pm 0.71$ & $-7.93 \pm 0.6$ \\
\hline J033330.4+311050 & $2.62 \pm 0.29$ & $-2.0 \pm 0.52$ & $-3.29 \pm 0.34$ \\
\hline J033346.9+305350 & $3.67 \pm 0.2$ & $10.92 \pm 0.31$ & $-12.58 \pm 0.2$ \\
\hline J033915.8+312430 & $2.37 \pm 0.7$ & $7.58 \pm 1.35$ & $-6.49 \pm 0.81$ \\
\hline
\end{tabular}




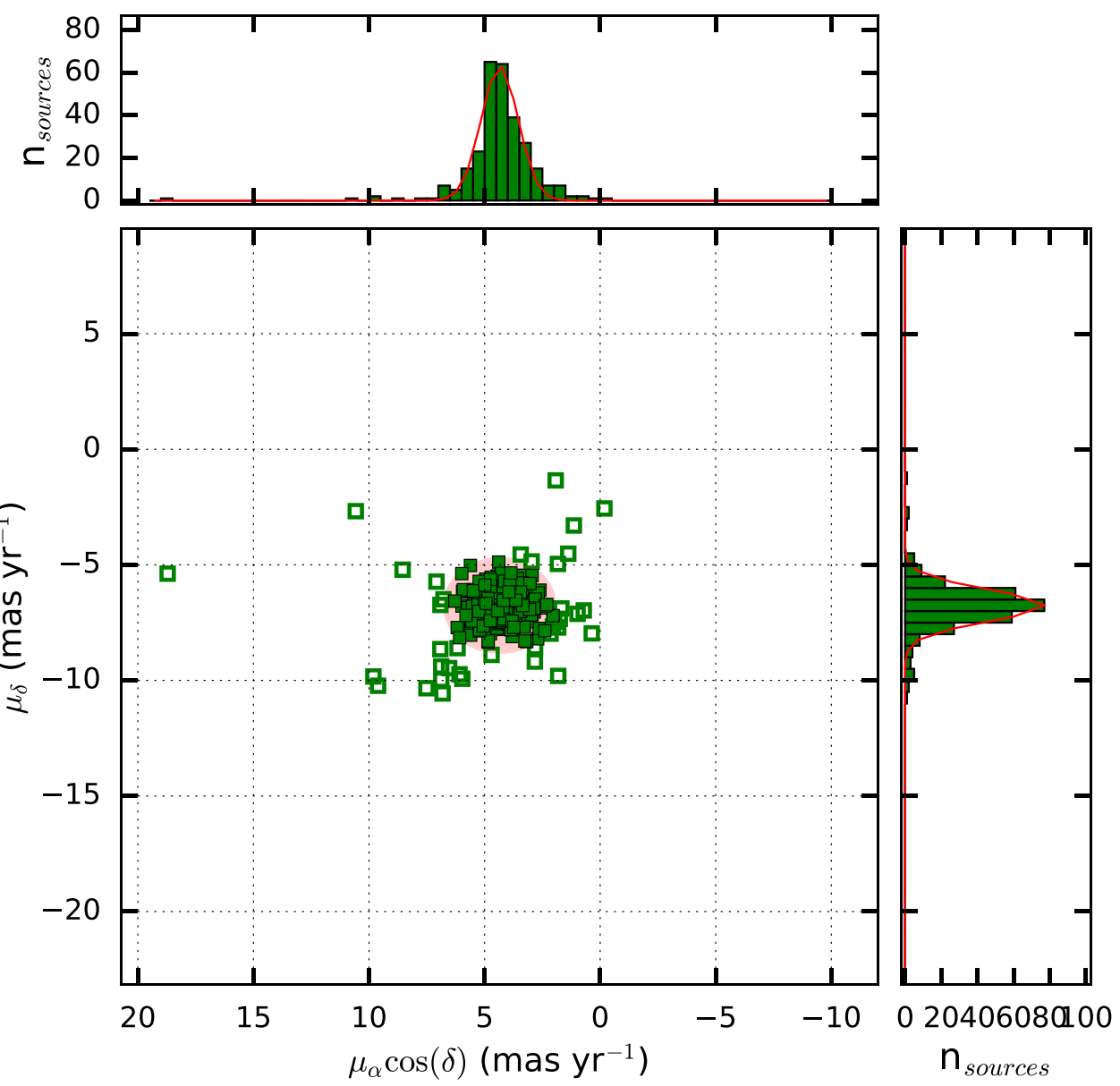

Figure 5. Proper motions measured by Gaia in IC 348. The top and right panels show the distributions of $\mu_{\alpha} \cos \delta$ and $\mu_{\delta}$, respectively. The Gaussian fits to these distributions are plotted in red. The filled and open squares are stars with proper motions within and outside $\pm 3 \sigma$ from the mean, respectively. The $\pm 3 \sigma$ range in proper motions is covered by the red shadow.

dominated by the intrinsic dispersion, but by the uncertainties on individual parallaxes. Thus, the true depth of the clouds cannot be extracted from these measurements.

The VLBA data alone suggest that NGC 1333 and IC 348 are located at similar distances. However, only the source IRAS $03260+3111$ in NGC 1333 was used for the present analysis. This source is a multiple system where the angular separation between the VLBA components is $\sim 0$ ".7. We do not expect that at such separation the orbital motion has a measurable effect on the motion of individual components over a timescale of a few years. The fit does not completely agree with the observations, though (top panel of Figure 1), so it is still possible that an additional and much closer unseen companion is present in the system. Regarding IC 348, the weighted mean of the VLBA parallaxes measured for V913 Per and V918 Per yields $321 \pm 10$ pc. This is in agreement with the weighted mean distance derived from Gaia parallaxes. We thus recommend using $321 \pm 10 \mathrm{pc}$ as the distance to IC 348 and $293 \pm 22$ pc for NGC 1333. Finally, we note that the binary system LRL 11 , located at a distance of $373 \pm 11$ pc, may not be part of Perseus but a background object projected in the direction of IC 348 .

Outside IC 348 and NGC 1333, we found Gaia parallaxes for 13 objects (see Figure 4 and Table 5), which are known YSO candidates in Perseus (Dunham et al. 2015). One object resides in the outskirts of L1448 and has a parallax of
$0.68 \pm 0.43$ mas, which means it is not part of Perseus. Two objects are in the outskirts of L1455, with parallaxes of $1.90 \pm 1.98$ mas and $3.62 \pm 0.14$ mas, respectively. While the former value does not provide any useful information due to its large uncertainty, the latter is consistent within $2 \sigma$ with the mean of parallaxes measured in NGC 1333. The next nine objects are projected in the direction of the cloud B1. They have a weighted mean parallax of $3.35 \pm 0.06$ mas with a standard deviation of 0.32 mas (corresponding to $296 \pm 28 \mathrm{pc}$ ), which is also consistent with the weighted mean parallax of NGC 1333. The last object is found southwest of IC 348 and has a parallax of $2.37 \pm 0.70$ mas. The large uncertainty of this measurement makes it difficult to claim the actual distance to this star.

Putting it all together, both Gaia and VLBA measurements suggest that the eastern edge of Perseus could be about $28 \mathrm{pc}$ farther than the western edge, which is a significantly smaller distance variation than previously thought (e.g., Hirota et al. 2011). Past measurements of parallaxes also using VLBI resulted in a distance of $235 \pm 18 \mathrm{pc}$ for NGC 1333 (Hirota et al. 2011). The difference between this measurement and that derived in this work is $2.6 \sigma$. We should note that the measurements by Hirota et al. (2011) were obtained from a fit to water masers, whose flux and positions showed time variability during the observing period of 6 months. The peak velocity of the water emission also suffered a drift of 


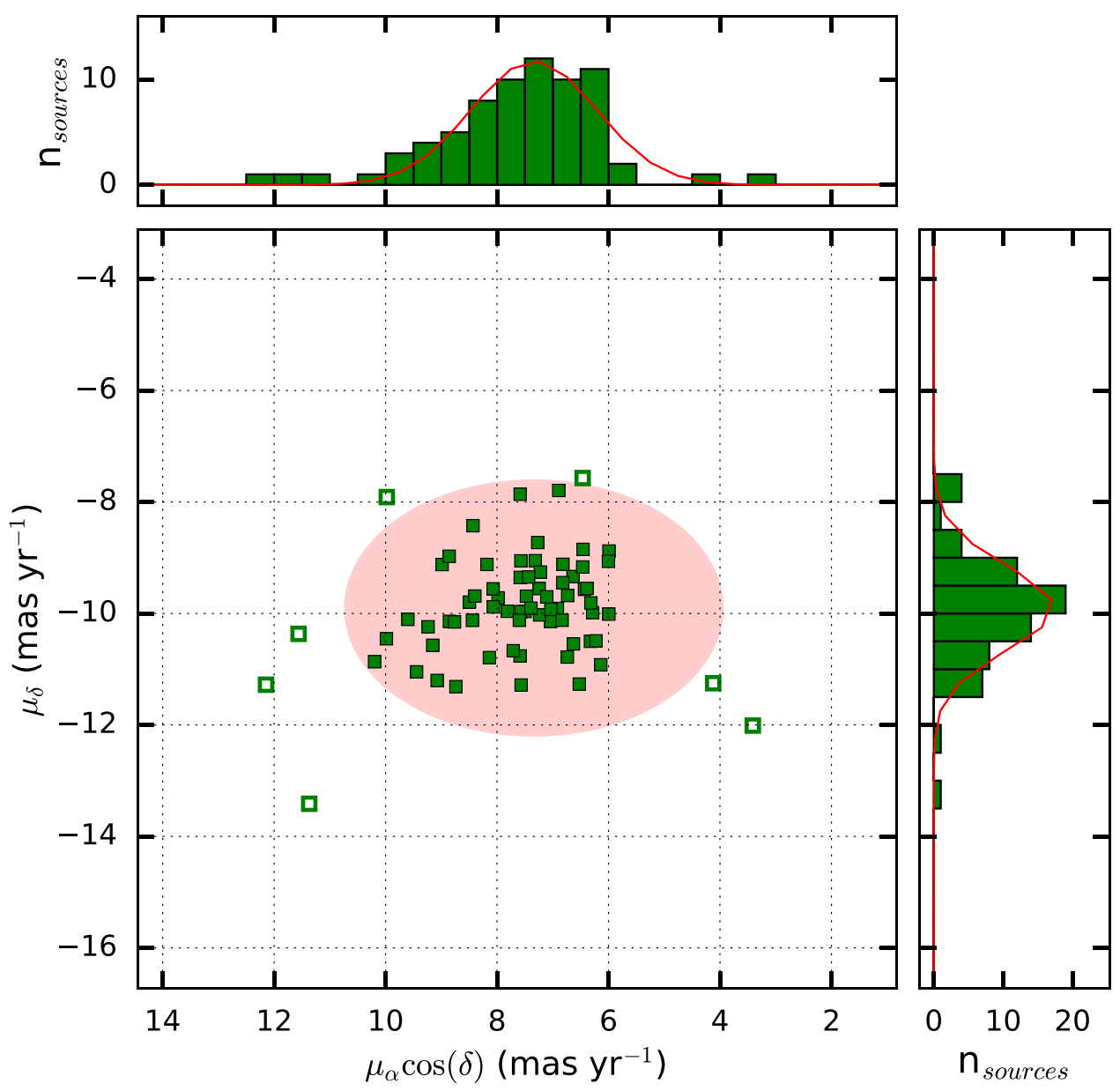

Figure 6. Same as Figure 5, but for NGC 1333.

$\sim 0.9 \mathrm{~km} \mathrm{~s}^{-1}$. These authors took the average position of the different maser "spots" (emission seen in a single velocity channel) over contiguous spectral channels, which resulted in two spatially separated "features" detected each during 3 and 4.5 months, respectively. We discussed in Dzib et al. (2018) that this approach can introduce position fluctuations larger than the synthesized beam size and can introduce additional uncertainty in the astrometric parameters of the masers. We demonstrated in Dzib et al. (2018) that, in order to reduce the chances of misidentifiying maser spots from one epoch to another, one should fit the maser positions measured at the same velocity channel in all epochs. In L1448, Hirota et al. (2011) also detected several spots at different velocity channels. In this case, the authors fit only maser spots detected at a velocity of $\sim 20.6 \mathrm{~km} \mathrm{~s}^{-1}$. However, their data span a baseline of only 5 months, which is not enough to properly cover the parallax sinusoid. It is possible that the variability of the maser emission led to a misidentification of the maser spots and affected the astrometry performed toward these masers. Unfortunately, the protostars to which these masers are associated are so embedded that they remained undetected by Gaia, so a direct comparison against Gaia astrometry is not possible at the moment. It is also possible that NGC 1333 has multiple components along the line of sight. However, as we mentioned above, the Gaia parallaxes are not good enough to search for such components.

\section{Kinematics of IC 348 and NGC 1333}

\subsection{Proper Motions}

To analyze the proper motions within NGC 1333 and IC 348 and their intrinsic velocity dispersion, we need first to define a subset of cluster members that reflect the true dynamics of the clusters. To construct such a sample, we exclude all sources with parallaxes that deviate by more than $3 \sigma$ from the weighted mean parallax in each cluster. The distributions of measured proper motions of the resulting sample after this initial cut are shown in Figures 5 and 6 for IC 348 and NGC 1333, respectively. The proper motion distributions were then fitted with Gaussian models, which are also plotted in red in these figures. We give in Table 6 the mean and velocity dispersion (corrected for the measurement errors) that result from the bestfit Gaussian distributions. To convert proper motion dispersions into tangential velocity dispersions, we used the mean distance of $321 \pm 10 \mathrm{pc}$ for IC 348 and $293 \pm 22 \mathrm{pc}$ for NGC 1333. Based solely on the analysis of the radial velocity distribution, Cottaar et al. (2015) measured a velocity dispersion of $0.72 \pm 0.07 \mathrm{~km} \mathrm{~s}^{-1}$ for IC 348. Similarly, Foster et al. (2015) measured $0.92 \pm 0.12 \mathrm{~km} \mathrm{~s}^{-1}$ for NGC 1333 . These values are comparable to the velocity dispersion measured here for the proper motions.

We then cut further stars with proper motions outside $\pm 3 \sigma$ from the mean, where $\sigma$ is the measured standard deviation. This selection has been made to mitigate the effects of 

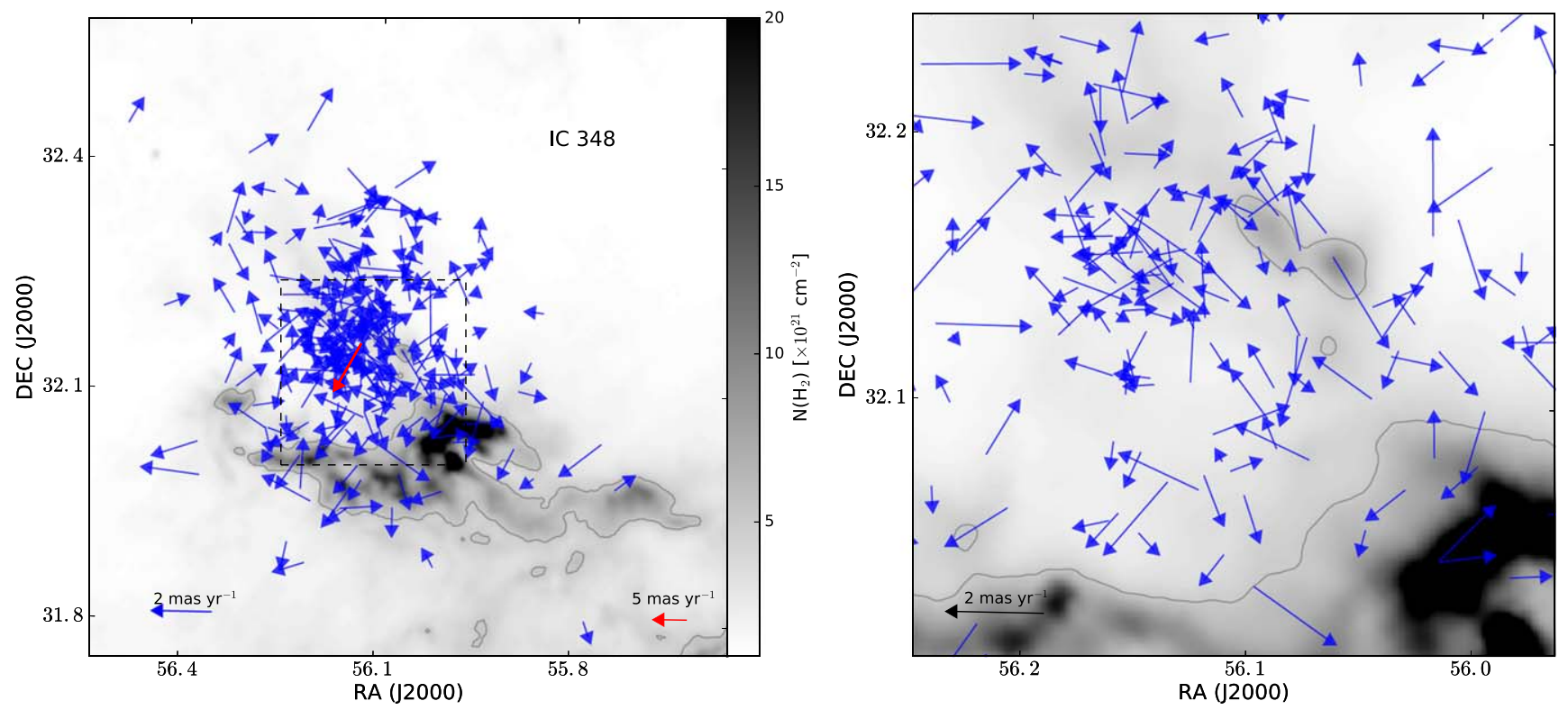

Figure 7. Measured proper motions by Gaia overlaid on the column density map derived from the Herschel Gould Belt survey (André et al. 2010) data by Sadavoy et al. (2014). The contour corresponds to $N\left(\mathrm{H}_{2}\right)=5 \times 10^{21} \mathrm{~cm}^{-2}$. The red arrow indicates the mean proper motion of the cluster obtained from a Gaussian fit to a subset of stars as described in Section 6.1. The blue arrows are individual measurements after subtracting the mean proper motion. The right panel shows a zoom-in of the central part of the left panel (dashed square).

unresolved astrometric binaries within our samples. Orbital motions are expected to contribute to the dispersion of the proper motions distributions in a nonpreferential orientation. In Figure 5, the stars that are cut by this criteria are plotted as green open squares, while the green filled squares represent the clusters members used in the forthcoming analysis. The proper motions of these subsets, relative to the mean of each cluster, are displayed in Figures 7 and 8, while the measured values are listed in Table 7. We see that proper motions within each cluster are highly consistent between themselves, with mean magnitudes indicated by the red arrows in each figure and given in Table 8 . Because these proper motions are measured relative to Sun, they mostly trace the reflex motion of the Sun. We must thus remove the solar motion for the analysis of the internal kinematics of the stars in Perseus.

\subsection{Spatial Velocities}

We now compute the three-dimensional Galactic spatial velocities of the reduced sample of stars described in the section above. This requires the conversion of proper motions and radial velocities into velocities in the rectangular system of Galactic coordinates $(x, y, z)$ where the Sun is at the origin.

Radial velocities (RVs) are available in the literature for several of our analyzed sources, which were obtained as part of the INfrared Spectra of Young Nebulous Clusters (IN-SYNC) ancillary program of the Apache Point Observatory Galactic Evolution Experiment (APOGEE) and published by Cottaar et al. (2014; for IC 348) and Foster et al. (2015; for NGC 1333). Kounkel et al. (2018) recently reported on a new reduction of the APOGEE data taken in Orion, IC 348, NGC 1333, and other regions. We use here the data products from this recent reduction since it implements an improved analysis of data variability. These RVs were measured at multiple epochs with typical baselines of a few months. Thus, we compute for each star the average of all available radial velocities, after removing epochs where the signal-to-noise ratio of the associated
Table 6

Derived Properties for IC 348 and NGC 1333

\begin{tabular}{lcc}
\hline \hline & IC 348 & NGC 1333 \\
\hline$\varpi_{\text {VLBA }}(\mathrm{mas})$ & $3.12 \pm 0.10$ & $\ldots$ \\
$\varpi_{\text {Gaia }}(\mathrm{mas})$ & $3.09 \pm 0.25$ & $3.38 \pm 0.26$ \\
$d_{\mathrm{VLBA}}(\mathrm{pc})$ & $321 \pm 10$ & $\ldots$ \\
$d_{\text {Gaia }}{ }^{\mathrm{a}}(\mathrm{pc})$ & $320_{-24}^{+28}$ & $293_{-21}^{+24}$ \\
$\bar{\mu}_{\alpha} \cos \delta\left(\mathrm{mas} \mathrm{yr}^{-1}\right)$ & $4.35 \pm 0.03$ & $7.34 \pm 0.05$ \\
$\bar{\mu}_{\delta}\left(\mathrm{mas} \mathrm{yr}^{-1}\right)$ & $-6.76 \pm 0.01$ & $-9.90 \pm 0.03$ \\
$\sigma_{\alpha}\left(\mathrm{mas} \mathrm{yr}^{-1}\right)$ & $0.24 \pm 0.03$ & $0.92 \pm 0.05$ \\
$\sigma_{\delta}\left(\mathrm{mas} \mathrm{yr}^{-1}\right)$ & $0.52 \pm 0.01$ & $0.60 \pm 0.03$ \\
$\sigma_{v_{\alpha}}\left(\mathrm{km} \mathrm{s}^{-1}\right)$ & $0.36 \pm 0.05$ & $1.27 \pm 0.07$ \\
$\sigma_{v \delta}\left(\mathrm{km} \mathrm{s}^{-1}\right)$ & $0.80 \pm 0.01$ & $0.83 \pm 0.04$ \\
$(\bar{X}, \bar{Y}, \bar{Z}) \mathrm{pc}^{-1}$ & $(-288,102,-98)$ & $(-255,101,-102)$ \\
$(\bar{U}, \bar{V}, \bar{W}) \mathrm{km} \mathrm{s}^{-1}$ & $(-17.2,-6.2,-8.2)$ & $(-17.5,-10.9,-9.6)$ \\
$(\bar{u}, \bar{v}, \bar{w}) \mathrm{km} \mathrm{s}^{-1}$ & $(-6.1,6.8,-0.9)$ & $(-6.4,2.1,-2.4)$ \\
$\left(\sigma_{u}, \sigma_{v}, \sigma_{w}\right) \mathrm{km} \mathrm{s}^{-1}$ & $(1.6,1.1,1.2)$ & $(1.0,1.4,1.0)$ \\
$v_{\text {exp }}\left(\mathrm{km} \mathrm{s}^{-1}\right)$ & -0.06 & 0.19 \\
$v_{\text {rot }}\left(\mathrm{km} \mathrm{s}^{-1}\right)$ & $(-0.16,0.0,-0.10)$ & $(-0.10,0.10,0.19)$ \\
\hline
\end{tabular}

Note.

${ }^{\text {a }}$ Corrected for the parallax zero-point shift of $-30 \mu$ as.

spectrum is less than 20 and the best-fit effective temperature is less than $2400 \mathrm{~K}$. As noted by Cottaar et al. (2015), such epochs do not provide useful RVs and should be discarded in our analysis. For this analysis, we selected stars with rotational velocities in the range $5-150 \mathrm{~km} \mathrm{~s}^{-1}$ (Cottaar et al. 2015) and RV uncertainties smaller than $2 \mathrm{~km} \mathrm{~s}^{-1}$ and excluded stars with very large proper motions, i.e., $>50 \mathrm{~km} \mathrm{~s}^{-1}$.

Furthermore, we removed stars with variable RVs, since epoch-to-epoch variations would be induced by binaries with short periods whose orbital motions would introduce a velocity offset. To look for strong radial velocity variability, we adopted the same procedure followed by Foster et al. (2015) in their own analysis of RVs. We computed the probability that the radial velocity is consistent with being constant, as estimated 
Table 7

Astrometric Parameters and Radial Velocities of Individual Sources in IC 348 and NGC 1333

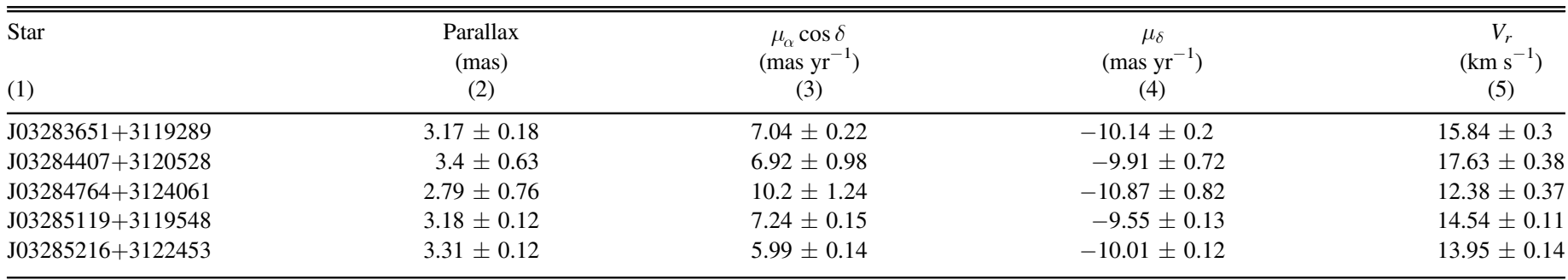

(This table is available in its entirety in machine-readable form.)

Table 8

Spatial Velocities and Positions of Individual Sources in IC 348 and NGC 1333

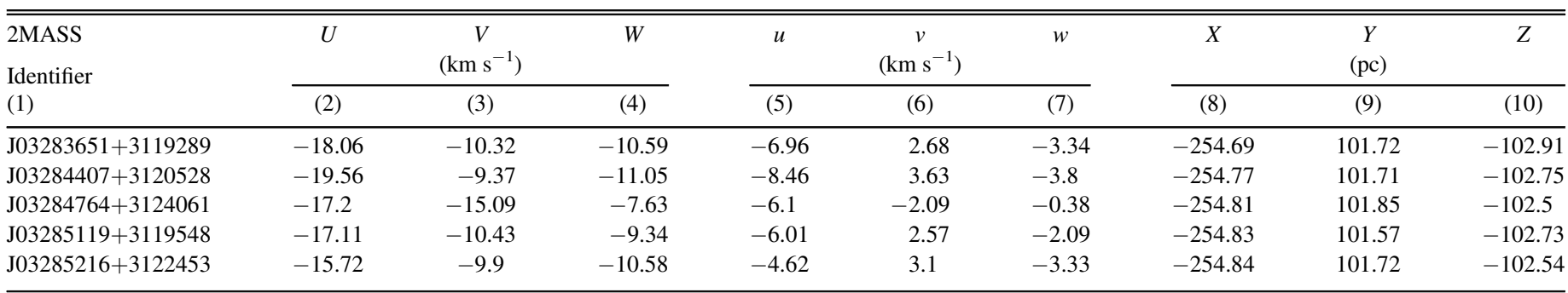

(This table is available in its entirety in machine-readable form.)

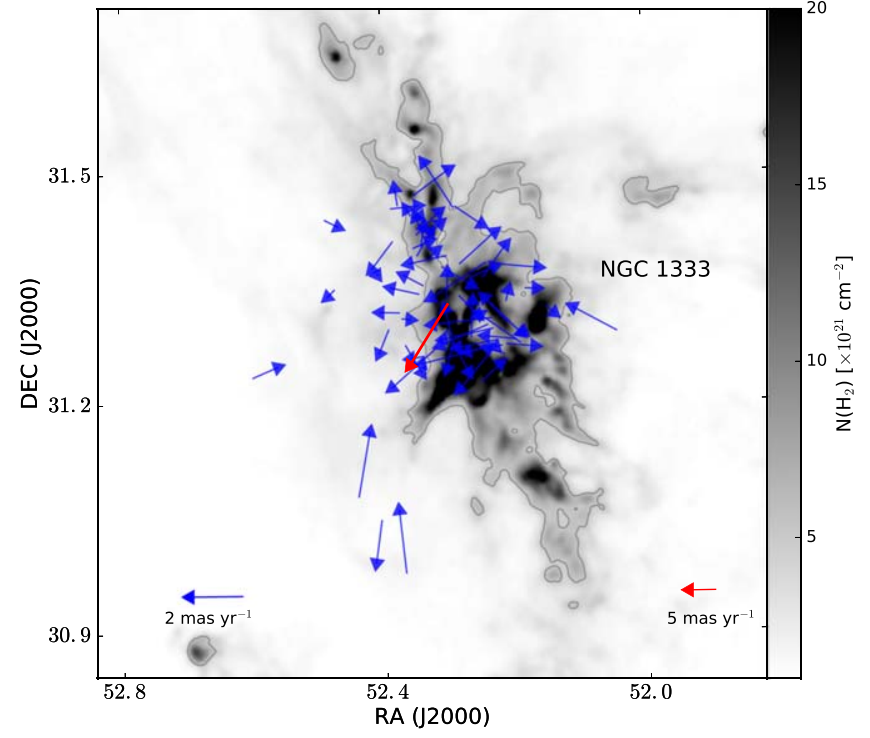

Figure 8. Same as Figure 7, but for NGC 1333.

from the $p$-value that the $\chi^{2}=\sum\left(\mathrm{RV}_{\mathrm{i}}-\mu\right)^{2} / \sigma_{i}^{2}$ is larger than expected from chance, where $\mathrm{RV}_{i}$ is the radial velocity with uncertainty $\sigma_{i}$ in epoch $i$ and $\mu$ is the weighted mean over all epochs. Following Foster et al. (2015), all sources with $p$-values smaller than $10^{-4}$ were excluded. The number of sources used to investigate the kinematics of the clouds, after removing the RV-variable sources, is 133 in IC 348 and 31 in NGC 1333. Their radial velocities are given in Table 7.

The velocities $(U, V, W)$ of each star relative to the $(x, y, z)$ reference system are listed in Table 7 . These were transformed to $(u, v, w)$ LSR velocities by subtracting the peculiar motion of the Sun, for which we use the values of the solar motion obtained by Schönrich et al. (2010): $U_{0}=11.1 \pm 0.7 \mathrm{~km} \mathrm{~s}^{-1}$, $V_{0}=12.2 \pm 0.47 \mathrm{~km} \mathrm{~s}^{-1}$, and $W_{0}=7.25 \pm 0.37 \mathrm{~km} \mathrm{~s}^{-1}$.

In the top panel of Figure 9, we show the projections of the mean LSR velocities $(\bar{u}, \bar{v}, \bar{w})$ as the blue and red arrows for IC 348 and NGC 1333, respectively. We found $(\bar{U}, \bar{V}, \bar{W})_{\text {IC } 348}=(-17.2 \pm 1.6,-6.2 \pm 1.1,-8.2 \pm$ $1.2) \mathrm{km} \mathrm{s}^{-1},(\bar{u}, \bar{v}, \bar{w})_{\text {IC } 348}=(-6.1 \pm 1.6,6.8 \pm 1.1,-0.9 \pm$ $1.2) \mathrm{km} \mathrm{s}^{-1},(\bar{U}, \bar{V}, \bar{W})_{\mathrm{NGC}} 1333=(-17.5 \pm 1.0,-10.9 \pm 1.4$, $-9.6 \pm 1.0) \mathrm{km} \mathrm{s}^{-1}$, and $(\bar{u}, \bar{v}, \bar{w})_{\mathrm{NGC}} 1333=(-6.4 \pm 1.0$, $2.1 \pm 1.4,-2.4 \pm 1.0) \mathrm{km} \mathrm{s}^{-1}$, where the quoted errors correspond to the standard deviation. For the calculation of these spatial velocity components, we have used the average distances derived in Section 5 because, as we have already pointed out, the individual parallax uncertainties are large (i.e., comparable to the parallax dispersion) that would broaden the velocity dispersion. The resulting 3D velocity dispersion is $\sigma=\sqrt{\sigma_{u}^{2}+\sigma_{v}^{2}+\sigma_{w}^{2}}=2 \mathrm{~km} \mathrm{~s}^{-1}$ for both clusters.

There is a significant difference between the velocity vectors $(\bar{U}, \bar{W}, \bar{W})$ measured here and those measured for the Perseus OB2 association, which overlaps the Perseus molecular cloud in the sky. On the basis of Hipparcos proper motions, Belikov et al. (2002) found $(U, W, W)=$ $(-12.7 \pm 1.6,-3.0 \pm 0.6,-0.9 \pm 0.8) \mathrm{km} \mathrm{s}^{-1}$ and a distance of $\sim 300 \mathrm{pc}$ for the association. This is not surprising given that the Perseus OB2 association, with an age of $6 \mathrm{Myr}$ (de Zeeuw et al. 1999), is older than both IC 348 and NGC 1333, and its dynamics has thus been affected by its interaction with the interstellar medium.

To calculate the expansion (or contraction) and rotation velocities within each cluster, we use the same methodology as 

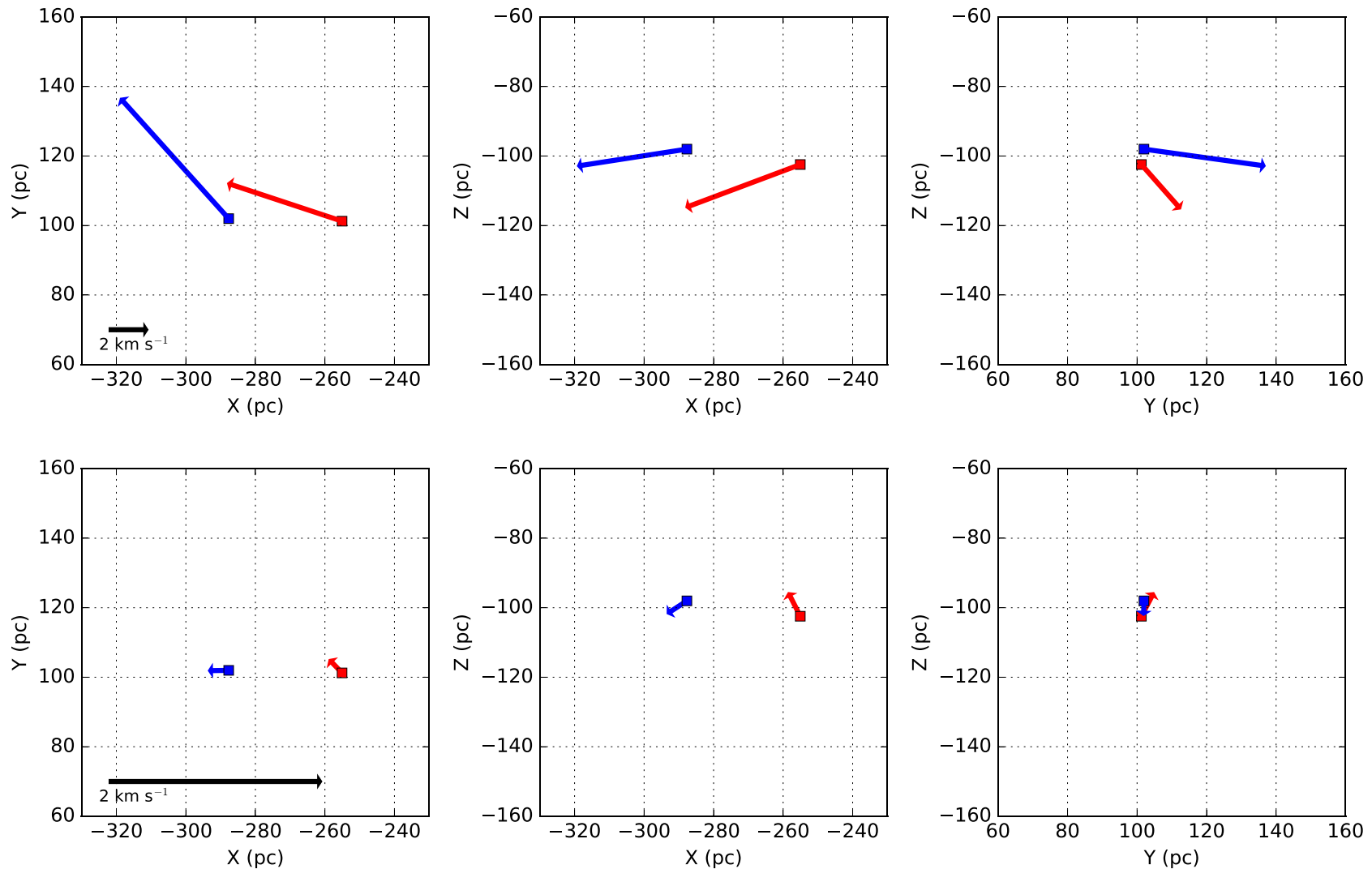

Figure 9. Top: mean of LSR velocities of stars in IC 348 (blue) and NGC 1333 (red) expressed in the rectangular system of Galactic coordinates. The origin of the arrows coincides with the mean of the $(X, Y, Z)$ positions of the stars in each cluster. Bottom: mean of the cross products $\hat{\boldsymbol{r}}_{*} \times \delta \boldsymbol{v}_{*}$ of stars in IC 348 (blue) and NGC 1333 (red). These vectors have been augmented by $6 \times$ for better visualization.

was used for the Taurus complex in Rivera et al. (2015). The expansion (or contraction) and rotation velocities are approximately given by the dot and cross products according to

$$
\begin{gathered}
v_{\exp }=\hat{\boldsymbol{r}}_{*} \cdot \boldsymbol{\delta} v_{*}, \\
v_{\text {rot }}=\hat{\boldsymbol{r}}_{*} \times \boldsymbol{\delta} v_{*},
\end{gathered}
$$

where $\hat{\boldsymbol{r}}_{*}=r_{*} /\left|r_{*}\right|$ is the unit vector of the position of the star relative to the cluster center and $\delta v_{*}$ is the velocity of the star with respect to the cluster itself.

These expansion and rotation velocities were computed for each star in our analyzed sample, and then we take the mean of each cluster to arrive at $v_{\text {exp,IC } 348}=-0.06 \mathrm{~km} \mathrm{~s}^{-1}$ and

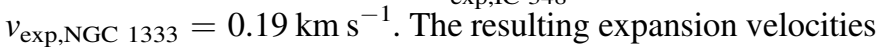
are very small compared with the velocity dispersion of $2 \mathrm{~km} \mathrm{~s}^{-1}$. This means that the stellar motions in the radial direction do not seem to follow an expansion or contraction pattern.

The bottom panel of Figure 9 shows the projection of the mean rotation velocities. $v_{\text {rot,IC } 348}=(-0.16,0.0,-0.10) \mathrm{km} \mathrm{s}^{-1}$, and $v_{\text {rot,NGC } 1333}=(-0.10,0.10,0.19) \mathrm{km} \mathrm{s}^{-1}$. These measurements suggest that the rotation velocity of both clusters is too small, if present at all.

In IC 348, Cottaar et al. (2015) found a velocity gradient of $0.024 \pm 0.013 \mathrm{~km} \mathrm{~s}^{-1} \operatorname{arcmin}^{-1}$ due to a possible solid-body rotation of the cluster. Since the region under consideration has a size of $\sim 36$ arcmin (Figure 6), this velocity gradient would imply a rotation velocity of $\sim 0.9 \pm 0.5 \mathrm{~km} \mathrm{~s}^{-1}$. Thus, the analysis presented here does not support the findings of Cottaar et al. (2015). It should be noted, moreover, that the statistical significance of that measurement is at the $1.8 \sigma$ level.

\section{Conclusions}

We have performed multi-epoch VLBA observations of three objects embedded in IC 348 and one object in NGC 1333, which are located near opposite ends within the Perseus molecular cloud. From the astrometric fits of this sample we derived a mean distance of $321 \pm 10 \mathrm{pc}$ to IC 348 , representing the most reliable distance determination to the eastern edge of Perseus. This distance is consistent with the mean of Gaia DR2 parallaxes to a selected sample of known, confirmed members of the cluster. The uncertainty on the mean of Gaia parallaxes is, however, 2.6 times larger than the VLBA uncertainty. The source detected with the VLBA in NGC 1333 is a close binary system, for which we derive preliminary orbital parameters. Unfortunately, the VLBA data are not enough to provide a reliable distance for this specific source and, consequently, for the NGC 1333 cluster. Gaia parallaxes, on the other hand, yield a mean distance of $293 \pm 22$ pc. From these measurements, we conclude that the distance between the western and eastern edges of the clouds is about $30 \mathrm{pc}$ in the direction of the line of sight.

We use Gaia proper motions and radial velocities from the literature to derive the spatial velocities for a subset of cluster members. We derive the average spatial velocity vectors of IC 348 and NGC 1333, which are similar in the magnitude and direction between them, but significantly different to the mean spatial motion of the Perseus OB2 association. We have estimated the expansion (or contraction) and rotation velocities of each cluster and found no clear evidence of such organized motions.

G.N.O.-L. acknowledges support from the von Humboldt Stiftung. P.A.B.G. acknowledges financial support from the 
São Paulo Research Foundation (FAPESP) through grants 2013/04934-8 and 2015/14696-2. L.L. acknowledges the financial support of DGAPA, UNAM (project IN112417), and CONACyT, México.

The Long Baseline Observatory is a facility of the National Science Foundation operated under cooperative agreement by Associated Universities, Inc. The National Radio Astronomy Observatory is a facility of the National Science Foundation operated under cooperative agreement by Associated Universities, Inc.

This work has made use of data from the European Space Agency (ESA) mission Gaia (https://www.cosmos.esa.int/ gaia), processed by the Gaia Data Processing and Analysis Consortium (DPAC, https://www.cosmos.esa.int/web/gaia/ dpac/consortium). Funding for the DPAC has been provided by national institutions, in particular the institutions participating in the Gaia Multilateral Agreement.

Funding for SDSS-III has been provided by the Alfred P. Sloan Foundation, the Participating Institutions, the National Science Foundation, and the U.S. Department of Energy Office of Science. The SDSS-III website is http://www.sdss3.org/. SDSS-III is managed by the Astrophysical Research Consortium for the Participating Institutions of the SDSS-III Collaboration including the University of Arizona, the Brazilian Participation Group, Brookhaven National Laboratory, Carnegie Mellon University, University of Florida, the French Participation Group, the German Participation Group, Harvard University, the Instituto de Astrofisica de Canarias, the Michigan State/Notre Dame/JINA Participation Group, Johns Hopkins University, Lawrence Berkeley National Laboratory, Max Planck Institute for Astrophysics, Max Planck Institute for Extraterrestrial Physics, New Mexico State University, New York University, Ohio State University, Pennsylvania State University, University of Portsmouth, Princeton University, the Spanish Participation Group, University of Tokyo, University of Utah, Vanderbilt University, University of Virginia, University of Washington, and Yale University.

\section{ORCID iDs}

Gisela N. Ortiz-León (1) https://orcid.org/0000-0002-2863-676X Laurent Loinard (1) https://orcid.org/0000-0002-5635-3345 Sergio A. Dzib (1) https://orcid.org/0000-0001-6010-6200 Phillip A. B. Galli (1) https://orcid.org/0000-0003-2271-9297 Marina Kounkel 1 ib https://orcid.org/0000-0002-5365-1267

Luis F. Rodríguez (i) https://orcid.org/0000-0003-2737-5681 Lee Hartmann (1) https://orcid.org/0000-0003-1430-8519 Cesar Briceño 누 https://orcid.org/0000-0001-7124-4094 John J. Tobin (1) https://orcid.org/0000-0002-6195-0152

\section{References}

Alexander, F., \& Preibisch, T. 2012, A\&A, 539, A64

Alves de Oliveira, C., Moraux, E., Bouvier, J., et al. 2013, A\&A, 549, A123

André, P., Men'shchikov, A., Bontemps, S., et al. 2010, A\&A, 518, L102

Bailer-Jones, C. A. L., Rybizki, J., Fouesneau, M., Mantelet, G., \& Andrae, R. 2018, AJ, 156, 58

Bally, J., Walawender, J., Johnstone, D., Kirk, H., \& Goodman, A. 2008, in Handbook of Star Forming Regions, Volume I: The Northern Sky, Vol. 4, ed. B. Reipurth (San Francisco, CA: ASP), 308

Belikov, A. N., Kharchenko, N. V., Piskunov, A. E., Schilbach, E., \& Scholz, R.-D. 2002, A\&A, 387, 117

Cernis, K. 1990, Ap\&SS, 166, 315

Cernis, K. 1993, BaltA, 2, 214

Connelley, M. S., Reipurth, B., \& Tokunaga, A. T. 2008, AJ, 135, 2496

Cottaar, M., Covey, K. R., Foster, J. B., et al. 2015, ApJ, 807, 27

Cottaar, M., Covey, K. R., Meyer, M. R., et al. 2014, ApJ, 794, 125

de Zeeuw, P. T., Hoogerwerf, R., de Bruijne, J. H. J., Brown, A. G. A., \& Blaauw, A. 1999, AJ, 117, 354

Dunham, M. M., Allen, L. E., Evans, N. J., II, et al. 2015, ApJS, 220, 11

Dzib, S. A., Ortiz-León, G. N., Hernández-Gómez, A., et al. 2018, A\&A, 614, A20

Enoch, M. L., Evans, N. J., II, Sargent, A. I., \& Glenn, J. 2009, ApJ, 692, 973

Foster, J. B., Cottaar, M., Covey, K. R., et al. 2015, ApJ, 799, 136

Gaia Collaboration, Brown, A. G. A., Vallenari, A., et al. 2018, A\&A, 616, A1

Gaia Collaboration, Prusti, T., de Bruijne, J. H. J., et al. 2016, A\&A, 595, A1

Galli, P. A. B., Loinard, L., Ortiz-Léon, G. N., et al. 2018, ApJ, 859, 33

Greisen, E. W. 2003, in Information Handling in Astronomy: Historical Vistas, Vol. 285, ed. A. Heck (New York: Springer), 109

Gutermuth, R. A., Myers, P. C., Megeath, S. T., et al. 2008, ApJ, 674, 336

Haisch, K. E., Jr., Greene, T. P., Barsony, M., \& Stahler, S. W. 2004, AJ, 127, 1747

Hirota, T., Bushimata, T., Choi, Y. K., et al. 2008, PASJ, 60, 37

Hirota, T., Honma, M., Imai, H., et al. 2011, PASJ, 63, 1

Kounkel, M., Covey, K., Suarez, G., et al. 2018, AJ, 156, 84

Kounkel, M., Hartmann, L., Loinard, L., et al. 2017, ApJ, 834, 142

Lindegren, L., Hernandez, J., Bombrun, A., et al. 2018, A\&A, 616, A2

Lombardi, M., Lada, C. J., \& Alves, J. 2010, A\&A, 512, A67

Luhman, K. L., Esplin, T. L., \& Loutrel, N. P. 2016, ApJ, 827, 52

Luri, X., Brown, A. G. A., Sarro, L. M., et al. 2018, A\&A, 616, A9

Ortiz-León, G. N., Dzib, S. A., Kounkel, M. A., et al. 2017a, ApJ, 834, 143

Ortiz-León, G. N., Loinard, L., Kounkel, M. A., et al. 2017b, ApJ, 834, 141

Pech, G., Loinard, L., Dzib, S. A., et al. 2016, ApJ, 818, 116

Rivera, J. L., Loinard, L., Dzib, S. A., et al. 2015, ApJ, 807, 119

Sadavoy, S. I., Di Francesco, J., André, P., et al. 2014, ApJL, 787, L18

Sadavoy, S. I., Di Francesco, J., Bontemps, S., et al. 2010, ApJ, 710, 1247

Schlafly, E. F., Green, G., Finkbeiner, D. P., et al. 2014, ApJ, 786, 29

Schönrich, R., Binney, J., \& Dehnen, W. 2010, MNRAS, 403, 1829

Tobin, J. J., Looney, L. W., Li, Z.-Y., et al. 2016, ApJ, 818, 73

Tychoniec, Ł., Tobin, J. J., Karska, A., et al. 2018, ApJ, 852, 18

Young, K. E., Young, C. H., Lai, S.-P., Dunham, M. M., \& Evans, N. J., II 2015, AJ, 150, 40 